\title{
Erratum to: Pravčice Rock Arch (Bohemian Switzerland National Park, Czech Republic) deterioration due to natural and anthropogenic weathering
}

Zuzana Vařilová $\cdot$ Richard Přikryl • Václav Cílek

Published online: 9 September 2011

(C) Springer-Verlag 2011

Erratum to: Environ Earth Sci (2011) 63:1861-1878

DOI 10.1007/s12665-010-0881-z

Unfortunately, some of the geographic exposures were incorrect in Tables 1,2 and 3. The correct versions of Tables 1, 2 and 3 are given below:

The online version of the original article can be found under doi:10.1007/s12665-010-0881-z.

Z. Vařilová

Bohemian Switzerland National Park Administration,

Krasna Lipa, Czech Republic

R. Přikryl ( $\bowtie)$

Institute of Geochemistry, Mineralogy and Mineral Resources,

Faculty of Science, Charles University in Prague, Albertov 6,

12843 Prague 2, Czech Republic

e-mail: prikryl@natur.cuni.cz

V. Cílek

Institute of Geology, Academy of Sciences of the Czech

Republic, Prague, Czech Republic 
Table 1 List of samples taken from the PRA and subjected to analytical study

\begin{tabular}{|c|c|c|}
\hline Sample & Height level (m) & Description \\
\hline \multicolumn{3}{|c|}{ Profile I, height level A (base of lower part), western exposure } \\
\hline I/A/1 & $0.5-0.9$ & White to grey salt efflorescence \\
\hline I/A/2 & $0.5-0.9$ & Dendritic grey efflorescence \\
\hline $\mathrm{I} / \mathrm{B}$ & $1.5-1.7$ & White to light grey isolated efflorescence \\
\hline $\mathrm{I} / \mathrm{C}$ & $>1.7$ & White to grey isolated efflorescence in cavities \\
\hline $\mathrm{I} / \mathrm{D}$ & $1.3-1.8$ & White to ochre, crumbling efflorescence \\
\hline \multicolumn{3}{|c|}{ Profile II, height level A (base of lower part), southern exposure (southern pillar of the PRA) } \\
\hline II/A & 0.5 & Compact thin whitish to light grey efflorescence \\
\hline II/B & $1.6-2.0$ & Dendritic white efflorescence \\
\hline \multicolumn{3}{|c|}{ Profile III, height level A (base of medium part), eastern exposure (southern pillar of the PRA) } \\
\hline III/A/1 & 0.3 & Light grey to grey efflorescence on blackened case-hardened surface \\
\hline III/A/2 & $0.8-1.0$ & Efflorescence on exposed (disintegrated) sandstone \\
\hline $\mathrm{III} / \mathrm{B} / 1$ & $0.0-0.3$ & Grey salt efflorescence \\
\hline $\mathrm{III} / \mathrm{B} / 2$ & $0.4-1.8$ & Light grey to grey efflorescence on blackened case-hardened surface \\
\hline $\mathrm{III} / \mathrm{B} / 3$ & $1.8-2.4$ & White to light grey efflorescence on the rims of pits \\
\hline \multicolumn{3}{|c|}{ Profile IV, height level A (base of medium part), south-western exposure (northern pillar of the PRA) } \\
\hline IV/1 & 1.8 & Continuous brownish efflorescence on exposed (disintegrated) sandstone \\
\hline $\mathrm{IV} / 2$ & 3.0 & Light grey to grey efflorescence on blackened case-hardened surface \\
\hline \multicolumn{3}{|c|}{ Profile V, height level B (base of medium part), south-eastern exposure (southern pillar of the PRA) } \\
\hline $\mathrm{V} / 1$ & $0.0-0.05$ & Continuous and dendritic whitish to ochre efflorescence \\
\hline $\mathrm{V} / 2$ & $0.05-0.2$ & Dendritic grey efflorescence \\
\hline $\mathrm{V} / 3$ & 0.2 & White to light grey "globules" \\
\hline $\mathrm{V} / 4$ & $0.3-0.5$ & Dendritic light grey efflorescence \\
\hline $\mathrm{V} / 5$ & $0.9-1.3$ & Dendritic light grey efflorescence \\
\hline $\mathrm{V} / 6$ & $1.4-1.6$ & Dendritic light grey efflorescence \\
\hline $\mathrm{V} / 7$ & $1.9-2.0$ & Dendritic light grey efflorescence \\
\hline \multicolumn{3}{|c|}{ Profile VI, height level $\mathrm{C}$ (close to the base), eastern exposure } \\
\hline $\mathrm{VI} / 1$ & $0.0-0.8$ & Continuous whitish efflorescence \\
\hline $\mathrm{VI} / 2$ & $0.8-1.8$ & Continuous whitish efflorescence \\
\hline
\end{tabular}


Table 2 Crystalline phases as determined by XRD in salt efflorescence from the PRA

\begin{tabular}{lllllll}
\hline Sample & Height level $(\mathrm{m})$ & Sulphates & Nitrates & Chlorides & Carbonates & $\begin{array}{l}\text { Mixed } \\
\text { salts }\end{array}$ \\
\hline
\end{tabular}

Profile III, height level A (base of medium part), eastern exposure (southern pillar of the PRA)

\begin{tabular}{|c|c|c|c|c|}
\hline $\mathrm{III} / \mathrm{B} / 3$ & $1.8-2.4$ & $\mathrm{~K}-\mathrm{al}(+++)$ & & hyd $( \pm)$ \\
\hline $\mathrm{III} / \mathrm{B} / 2$ & $0.4-1.8$ & gyp $(+++)$, tsch $(+)$, al $( \pm)$ & nit $(+)$ & \\
\hline $\mathrm{III} / \mathrm{A} / 2$ & $0.8-1.0$ & K-al $(+++)$, gyp $(+)$ & & hyd $( \pm)$ \\
\hline III/A/1 & 0.3 & $\operatorname{gyp}(++), \operatorname{tsch}(+)$ & amnit $(+)$ & krem $(+)$ \\
\hline $\mathrm{III} / \mathrm{B} / 1$ & $0.0-0.3$ & $\operatorname{gyp}(+++), \operatorname{tsch}(++)$, al $(+)$ & & \\
\hline
\end{tabular}

Profile IV, height level A (base of medium part), south-western exposure (northern pillar of the PRA)
$\mathrm{IV} / 2 \quad 3.0$
gyp $(+++), \mathrm{Na}-\mathrm{al}(+)$
sal $( \pm)$
$\mathrm{IV} / 1 \quad 1.8$
gyp $(++), \mathrm{K}-\mathrm{al}(+)$
hyd $(+), \sin ( \pm)$
ox $( \pm)$

Profile I, height level A (base of lower part), western exposure

$\begin{array}{llllll}\text { I/D } & 1.3-1.8 & \text { K-al }(+++), \text { gyp }(+) & & \text { hl }( \pm), \text { hyd }( \pm) & \\ \text { I/C } & >1.7 & \text { gyp }(++), \text { tsch }(+), \text { K-al }(+), \text { Na-al }( \pm) & \operatorname{nitr}(++) & & \text { plut }(+) \\ \text { I/B } & 1.5-1.7 & \text { K-al }(+++), \text { gyp }(+) & \text { nitrm }(+) & & \text { kai }( \pm) \\ \text { I/A/2 } & 0.5-0.9 & \text { K-al }(+++), \text { gyp }(+) & \text { nitrm }(+) & \text { hyd }( \pm) & \text { nitr }(++) \\ \text { I/A/1 } & 0.5-0.9 & \text { K-al }(++), \text { gyp }(+), \text { al }(+) & & \end{array}$

Profile II, height level A (base of lower part), southern exposure (southern pillar of the PRA)

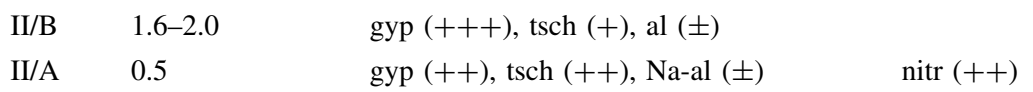

Profile V, height level B (base of medium part), south-eastern exposure (southern pillar of the PRA)

\begin{tabular}{|c|c|c|c|c|c|}
\hline $\mathrm{V} / 7$ & $1.9-2.0$ & gyp $(++), \mathrm{K}-\mathrm{al}(+)$, star $(+)$ & \multicolumn{3}{|c|}{ nah $( \pm)$} \\
\hline $\mathrm{V} / 6$ & $1.4-1.6$ & $\operatorname{gyp}(++), \mathrm{K}-\mathrm{al}(++)$ & hyd $(+)$ & & ox $(+)$ \\
\hline $\mathrm{V} / 5$ & $0.9-1.3$ & gyp $(++), \mathrm{K}-\mathrm{al}(++)$, star $(+)$ & & & $\mathrm{ox}(+)$ \\
\hline $\mathrm{V} / 4$ & $0.3-0.5$ & gyp $(++), \mathrm{K}-\mathrm{al}(++)$, tut $(+)$ & & & \\
\hline $\mathrm{V} / 3$ & 0.2 & $\begin{array}{l}\text { hal }(++) \text {, alg }(+) \text {, alm }(+) \text { Na-al }(+) \text {, } \\
\quad \text { eps }(+), \text { mel }( \pm), \text { syn }( \pm)\end{array}$ & $\sin ( \pm)$ & gor $( \pm)$ & \\
\hline $\mathrm{V} / 2$ & $0.05-0.2$ & K-al $(+++)$, gyp $(+)$ & hyd $( \pm)$ & & \\
\hline $\mathrm{V} / 1$ & $0.0-0.05$ & gyp $(+++), \mathrm{K}$-al $(++)$, alg $(+)$ & krem $(+)$ & & \\
\hline Profile & height lev & se to the base), eastern exposure & & & \\
\hline $\mathrm{VI} / 2$ & $0.8-1.8$ & $\mathrm{~K}-\mathrm{al}(+++), \operatorname{gyp}(+)$ & hyd $( \pm)$ & & \\
\hline $\mathrm{VI} / 1$ & $0.0-0.8$ & $\mathrm{~K}-\mathrm{al}(+++), \operatorname{gyp}(++)$ & hyd $( \pm)$ & & \\
\hline
\end{tabular}

The semiquantitative data are provided as +++ (above $50 \mathrm{wt} . \%$, dominant phase), $++(15-50 \mathrm{wt} . \%$, major phase $),+(5-15 \mathrm{wt} . \%$, minor phase), \pm (below 5 wt. $\%$, traces)

gyp gypsum, al alunite, tsch tschermigite, Na-al natroalunite, $\mathrm{K}$-al potassium alum, hal halotrichite, alg alunogen, alm alumogenite, syn syngenite, eps epsomite, mel melanterite, nitm nitrammite, nitr nitratine, nit niter, amnit ammonian niter, ha halite, hyd hydrophilite, sal salammoniac, sin sinjarite, krem kremersite, nah nahcolite, plut $\mathrm{B} /$ Plutschliite, gor gordaite, kai kainite, ox oxammite 
Table 3 Water-soluble salts content (in wt $\%$, determined by IEC) in efflorescence on sandstone of the PRA

\begin{tabular}{|c|c|c|c|c|c|c|c|c|c|}
\hline Sample & Height level (m) & $\mathrm{Cl}^{-}$ & $\mathrm{NO}_{3}{ }^{-}$ & $\mathrm{SO}_{4}{ }^{2-}$ & $\mathrm{Na}^{+}$ & $\mathrm{NH}_{4}^{+}$ & $\mathrm{K}^{+}$ & $\mathrm{Ca}^{2+}$ & $\mathrm{Mg}^{2+}$ \\
\hline \multicolumn{10}{|c|}{ Profile III, height level A (base of medium part), eastern exposure (southern pillar of the PRA) } \\
\hline III/A/1 & 0.3 & 0.10 & 0.10 & 26.50 & 0.40 & 1.90 & 0.50 & 1.10 & b.d.l. \\
\hline $\mathrm{III} / \mathrm{A} / 2$ & $0.8-1.0$ & b.d.1. & b.d.1. & 30.60 & 0.10 & 2.80 & 0.50 & 1.20 & b.d.1. \\
\hline III/B/1 & $0.0-0.3$ & 0.10 & 0.10 & 9.70 & 0.10 & 0.50 & 0.10 & 1.20 & b.d.l. \\
\hline $\mathrm{III} / \mathrm{B} / 2$ & $0.4-1.8$ & 0.02 & 0.10 & 3.20 & 0.04 & 0.20 & 0.10 & 0.40 & b.d.1. \\
\hline $\mathrm{III} / \mathrm{B} / 3$ & $1.8-2.4$ & 0.10 & 0.20 & 28.70 & 0.20 & 2.50 & 1.10 & 0.50 & b.d.l. \\
\hline \multicolumn{10}{|c|}{ Profile IV, height level A (base of medium part), south-western exposure (northern pillar of the PRA) } \\
\hline IV/1 & 1.8 & 0.10 & 0.01 & 20.00 & 0.20 & 1.80 & 1.20 & 0.70 & 0.10 \\
\hline $\mathrm{IV} / 2$ & 3.0 & 0.10 & 0.05 & 18.90 & 0.10 & 1.40 & 0.80 & 0.90 & 0.20 \\
\hline \multicolumn{10}{|c|}{ Profile I, height level A (base of lower part), western exposure } \\
\hline $\mathrm{I} / \mathrm{D}$ & $1.3-1.8$ & 0.02 & 0.10 & 23.20 & 0.10 & 1.30 & 2.30 & 0.50 & b.d.1. \\
\hline $\mathrm{I} / \mathrm{C}$ & $>1.7$ & 0.10 & 0.02 & 25.20 & 0.10 & 1.80 & 0.80 & 1.10 & b.d.l. \\
\hline $\mathrm{I} / \mathrm{B}$ & $1.5-1.7$ & 0.03 & 0.10 & 27.80 & 0.02 & 2.10 & 1.80 & 0.50 & b.d.l. \\
\hline $\mathrm{I} / \mathrm{A} / 2$ & $0.5-0.9$ & 0.20 & 0.30 & 25.10 & 0.10 & 1.60 & 1.80 & 0.50 & b.d.l. \\
\hline I/A/1 & $0.5-0.9$ & 2.70 & 2.60 & 19.80 & 0.10 & 1.00 & 0.60 & 2.60 & 0.10 \\
\hline \multicolumn{10}{|c|}{ Profile II, height level A (base of lower part), southern exposure (southern pillar of the PRA) } \\
\hline II/B & $1.6-2.0$ & 0.10 & b.d.1. & 10.50 & 0.10 & 0.50 & 0.30 & 0.80 & 0.03 \\
\hline II/A & 0.5 & 0.10 & 0.10 & 18.50 & 0.10 & 1.30 & 0.90 & 0.70 & b.d.1. \\
\hline \multicolumn{10}{|c|}{ Profile V, height level B (base of medium part), south-eastern exposure (southern pillar of the PRA) } \\
\hline $\mathrm{V} / 7$ & $1.9-2.0$ & 0.10 & b.d.l. & 19.80 & 0.20 & 1.10 & 1.50 & 0.90 & 0.02 \\
\hline V/6 & $1.4-1.6$ & 0.10 & b.d.l. & 21.40 & 0.20 & 1.2 & 1.60 & 1.30 & 0.02 \\
\hline $\mathrm{V} / 5$ & $0.9-1.3$ & 0.10 & b.d.l. & 18.70 & 0.20 & 1.00 & 1.20 & 0.90 & 0.02 \\
\hline $\mathrm{V} / 4$ & $0.3-0.5$ & 0.10 & b.d.l. & 16.20 & 0.20 & 1.10 & 1.30 & 1.00 & 0.10 \\
\hline V/3white & 0.2 & 0.20 & 0.05 & 40.50 & 0.50 & 0.01 & b.d.l. & b.d.l. & 1.50 \\
\hline V/3yellow & 0.2 & 0.20 & 0.04 & 37.70 & 0.20 & b.d.l. & 0.10 & 0.01 & 0.30 \\
\hline $\mathrm{V} / 2$ & $0.05-0.2$ & 0.10 & b.d.l. & 21.60 & 0.10 & 0.80 & 1.20 & 1.80 & b.d.l. \\
\hline $\mathrm{V} / 1$ & $0.0-0.05$ & 0.10 & b.d.l. & 20.00 & 0.10 & 0.90 & 1.20 & 1.00 & 0.10 \\
\hline \multicolumn{10}{|c|}{ Profile VI, height level C (close to the base), eastern exposure } \\
\hline $\mathrm{VI} / 2$ & $0.8-1.8$ & 0.10 & b.d.l. & 18.80 & 0.10 & 1.20 & 2.30 & 0.1 & b.d.l. \\
\hline $\mathrm{VI} / 1$ & $0.0-0.8$ & 0.10 & b.d.1. & 23.30 & 0.20 & 1.30 & 2.80 & 0.2 & b.d.1. \\
\hline
\end{tabular}

b.d.l. below detection limit (which is $0.001 \mathrm{wt} . \%$ for the method and species determined) 